\title{
ISOLATION OF ANAEROBES IN DEEP SEATED PRESSURE ULCERS USING A NOVEL INNOVATIVE TECHNIQUE OF ANAEROBE ISOLATION
}

\author{
Lalbiaktluangi1, Bipasa Chakraborty², Jayeeta Halder 3 , Prasanta Kumar Maiti4, Raja Ray5 \\ ${ }_{1}^{1}$ PGT (3rd year), Department of Microbiology, Institute of Post Graduate Medical Education and Research, Kolkata. \\ ${ }^{2}$ Demonstrator, Department of Microbiology, Burdwan Medical College and Hospital, Burdwan. \\ ${ }^{3} \mathrm{PhD}$ Scholar, Department of Microbiology, Institute of Post Graduate Medical Education and Research, Kolkata. \\ ${ }^{4}$ Professor and HOD, Department of Microbiology, Institute of Post Graduate Medical Education and Research, Kolkata. \\ ${ }^{5}$ Professor, Department of Microbiology, Institute of Post Graduate Medical Education and Research, Kolkata.
}

\section{ABSTRACT}

\section{BACKGROUND}

Isolation of an anaerobe is usually neglected in hospitals with limited resources due to the expensive and complicated technique of anaerobic isolation methods, which is difficult to arrange in such resource poor settings. Conventionally adopted anaerobic culture methods such as Anaerobic jar, Gas-Pak, Anoxomat or Automated glove-box systems are extremely costly and cumbersome for single unit testing, but not suitable for small scale laboratories. However, anaerobic bacteria are not to be overlooked as they have made a comeback in clinical settings and are even showing resistance to Metronidazole, once thought to be the gold standard bullet against anaerobes. Deep seated pressure ulcers are usually the site where anaerobe causes an infection in synergy with aerobes.

\section{AIMS AND OBJECTIVES}

Isolation of anaerobes in deep seated pressure ulcers using a novel innovative technique and to study their antibiogram profile.

\section{MATERIALS AND METHODS}

Swabs taken from depth of deep seated pressure ulcers were immediately inoculated in Brucella blood agar at bedside and placed in polycarbonate airtight jar for anaerobic incubation using a novel innovative Modified Candle Jar technique. In this technique five grams of grease-free grade zero steel wool were dipped in $50 \mathrm{ml}$ freshly prepared acidified copper sulphate solution until the copper colour appeared. Excess solution was drained and the steel wool was moulded into a loose pad to fit on an open Petri plate placed on top of the inoculated Brucella blood agar plates. A white-wax candle was placed at the centre of this plate. A small test tube containing mixture of $0.5 \mathrm{~g}$ sodium-bicarbonate and $0.5 \mathrm{~g}$ magnesium carbonate was kept ready to be placed inside the jar, just after placing the inoculated plate and incubated for 48 hours.

\section{RESULTS}

Peptostreptococcus anaerobius and Bacteroides fragilis were successfully isolated from deep seated pressure ulcers by this method. Antibiogram studies were done using the recommended E-test technique. In $50 \%$ cases of Bacteroides fragilis, resistance to Metronidazole was seen.

\section{CONCLUSION}

The advantage of this innovative technique of anaerobe isolation is, it is cost-effective and can be used in resource constrained settings and anaerobiosis is achieved at the bedside of the patient.

\section{KEYWORDS}

Bacteroides fragilis, Metronidazole Resistance, Modified Candle-Jar Technique, Peptostreptococcus anaerobius, Pressure Ulcers.

HOW TO CITE THIS ARTICLE: Lalbiaktluangi, Bipasa Chakraborty, Jayeeta Halder, Prasanta Kumar Maiti, Raja Ray. "Isolation of Anaerobes in Deep Seated Pressure Ulcers using a Novel Innovative Technique of Anaerobe Isolation." Journal of Evolution of Medical and Dental Sciences 2015; Vol. 4, Issue 98, December 07; Page: 16329-16333, DOI: 10.14260/jemds/2015/2411

\section{INTRODUCTION}

Anaerobes outnumber aerobes as a commensal flora in human body. They are found in the mucous membranes of oral cavity, upper respiratory passages, lower gastrointestinal tract, female genital tract and skin.[1] Non-sporing anaerobes are known to cause endogenous infections.[1,2] They cause infections in pressure ulcers along with aerobes with their clinical characteristic typical of a putrid and pervasive odour.

It may even result in complications like osteomyelitis and bacteraemia if not treated appropriately. The cause of infection is multifactorial and nature of involved organisms is

Financial or Other, Competing Interest: None.

Submission 19-11-2015, Peer Review 20-11-2015,

Acceptance 30-11-2015, Published 05-12-2015.

Corresponding Author:

Dr. Raja Ray,

A/11 Survey Park,

Santoshpur, Kolkata-700075.

E-mail: rjrm1175@gmail.com

DOI:10.14260/jemds/2015/2411 polymicrobial. Since anaerobes do not grow on solid media in ambient atmosphere $(0.04 \%$ carbon dioxide and $21 \%$ oxygen $)$, success for maximum isolation depends on maintenance of critically low oxygen levels and also a low redox potential and growth phase in a reduced medium with exclusion of inhibitory substances.

Hence, a dual system equipped with candle combustion for instant exhaustion of major part of oxygen from a sealed jar along with acidified steel wool for residual oxygen purging will be used as it is cost-effective, suitable for bed-side inoculation and processing. [3] Our method is merely but a modification or hybrid of the technique of Candle jar technique devised by Cruickshank in 1968 and Anaerobic bag culture method developed by Rosenblatt in 1975. Candle jar method was mainly used for cultivation of capnophilic organisms like Neisseria species, Haemophilus influenzae and Streptococcus pneumoniae since this technique produces additional (5-10\%) $\mathrm{CO}_{2}$ necessary for their growth, but there is no removal of 
residual $\mathrm{O}_{2 .}{ }^{[4]}$ Thus it is not suitable for isolation of obligate anaerobes.

Whereas in Rosenblatt's method of anaerobic culture, known as Anaerobic bag culture method, a transparent, gasimpermeable bag is used and the anaerobic environment is established with copper sulphate-saturated steel wool. An Alka-Seltzer tablet generates carbon dioxide. The agar plate surface can be inspected through the bag at any time without interrupting the anaerobic atmosphere or disturbing other specimens. Methylene blue indicator strips are completely reduced within $4 \mathrm{hrs}$. after the bag is set up and tend to remain reduced for as long as 3 weeks.[5] No consistent differences were found between the anaerobic bag and GasPak jar methods in the yield of anaerobes from clinical specimens.

Early growth is detected with the bag method when compared with GasPak. But the disadvantage of the anaerobic bag culture technique is that it has a small surface area due to which trapped air is present in between the petri dish lid and agar surface which leads to lesser recovery of obligate anaerobes.

Currently available methods like GasPak and Anoxomat are costly and cumbersome. That is why in most centres empirical treatment of anaerobic infections has become a routine practice. ${ }^{[6]}$ often resulting in irrational drug use, drug resistance development and fatal outcome. A simple costeffective method of anaerobiosis and technically simple antibiotic susceptibility testing may contribute towards rational treatment of such infections for resource limited centres.

\section{MATERIALS AND METHODS}

This study has been done after taking proper institutional ethical clearance. Deep seated pressure ulcers were selected in our study (Figure-1). Before swab was taken, the surrounding ulcer margins were cleaned, eschar and epithelialization were removed. Two swab from the depth of pressure ulcer site after superficial wound debridement with normal saline were taken. With one swab direct smear was prepared for gram staining and microscopy and the other swab was immediately inoculated directly in Brucella blood agar plates at the bedside of the patient and placed inside a polycarbonate jar with an airtight lid. Five grams of grease-free grade zero steel wool (Commercially available for scrubbing) was dipped in $50 \mathrm{ml}$ freshly prepared acidified copper sulphate solution (Mixture of $10 \%$ copper sulphate solution $5 \mathrm{ml}, 10 \%$ Tween $80 \mathrm{w} / \mathrm{v} 5 \mathrm{ml}$, Sulphuric acid (2mol/l) $3 \mathrm{ml}$ and distilled water up to $200 \mathrm{ml}$ ) until copper Colour appeared.

Excess solution was drained and the steel wool was moulded into a loose pad to fit on an open Petri plate placed on top of the inoculated Brucella blood agar plates. A two inches long white-wax candle was placed at the centre of this plate. A small test tube containing mixture of $0.5 \mathrm{~g}$ sodiumbicarbonate and $0.5 \mathrm{~g}$ magnesium carbonate was kept ready to put inside the jar just after placing inoculated plate.

This $1000 \mathrm{ml}$ jar holds three inoculated plates at a time while one $500 \mathrm{ml}$ jar could be used for single plate incubation (Figure-2). After 48 hours incubation at $37^{\circ} \mathrm{C}$ by this modified candle jar method, there was growth of organism. When subculture of the same colony for aero-tolerance testing was done, it showed no growth, which confirmed it to be anaerobe. Biochemical identification and antibiotic sensitivity testing by E-test strip. ${ }^{[7]}$ were also done in this same system and the isolated anaerobes were identified as Bacteroides fragilis and Peptostreptococcus anaerobius.

\section{RESULTS}

Out of the total 50 cases presenting with pressure ulcers, deep seated pressure ulcer were observed only in 8 cases, all of which showed polymicrobial infection. Out of these 8 cases, anaerobes were isolated in 6 cases. Bacteroides fragilis (4) and Peptostreptococcus anaerobius (2) were the anaerobes isolated. Direct smear for Bacteroides fragilis showed plenty of pus cells with few gram negative bacilli. Colony morphology on Brucella blood agar showed white to grey, circular, convex, translucent to semi opaque, nonhaemolytic colony with entire margin (Figure-3a), and in Bacteroides bile esculine (BBE) agar; colonies were larger than $1 \mathrm{~mm}$, circular, entire margin raised, low convex and black colonies (Figure-3b).

Gram staining appearance from the colony showed gram negative, pleomorphic rods with rounded ends. Aerotolerant test was found to be negative. The important biochemical identification tests were as follows- bile esculin hydrolysis positive; sugar fermentation tests for glucose, lactose and sucrose were positive; and maltose, arabinose and xylose were negative. Spot indole test and urease test were negative. The special potency disk test done for identification showed resistance for kanamycin $(1000 \mu \mathrm{g})$, Vancomycin $(5 \mu \mathrm{g})$ and colistin $(10 \mu \mathrm{g})$. Direct smear for Peptostreptococcus anaerobius showed plenty of pus cells with few gram positive cocci in singles, pairs and in short chains.

Culture on Brucella blood agar showed nonhaemolytic, grey white opaque colonies with sweet and foetid odour. Gram staining from culture showed gram positive large cocci in chains. Aerotolerant test was negative. The biochemical identification tests showed - spot indole test negative; fermentation of glucose, maltose positive; and no fermentation of lactose, sucrose and xylose was seen. Nitrate reduction test was negative, urease test was negative, coagulase was negative. For Peptostreptococcus anaerobius $(\mathrm{n}=2)$ paper discs impregnated with $5 \%$ sodium polyanethol sulphonate showed zone of inhibition of $17 \mathrm{~mm}$ and $18 \mathrm{~mm}$ each.

The special potency disk test done for identification showed sensitivity to Vancomycin $(5 \mu \mathrm{g})$ and resistance to kanamycin $(1000 \mu \mathrm{g})$ and colistin $(10 \mu \mathrm{g})$. Antibiotic sensitivity testing were done by E-test strips (Figure-4) and antibiogram profiles of all the 6 anaerobes were as shown in Table no. 1 . Interpretations as sensitive and resistant were done according to MIC interpretative criteria for anaerobes as given in CLSI guidelines 2015.[8] Metronidazole resistance was found in 2 isolates of Bacteroides fragilis. The isolated aerobes were Proteus mirabilis, Klebsiella species, Staphylococcus aureus, Providencia spp and Enterococcus faecalis.

\section{DISCUSSION}

Technical limitations of anaerobic culture arise mostly due to toxic oxygen exposure making isolation of anaerobes from clinical specimen quite difficult. Even with the anaerobic jar technique and anaerobic glove box system, the anaerobe isolation rates are low. ${ }^{[9,10]}$ For the large scale processing units such as the glove box system, transit time from collection point to the point of inoculation is long and oxygen exposure might occur during the transit. For smaller units such as the anaerobic jar, Gas Pak systems the time required for reduction of oxygen to the critically low levels $(0.7 \%)$ required for the culture of anaerobes is quite long and is usually more than 90 minutes. ${ }^{[5,11]}$ If this period exceeds the generation time of the anaerobes, this may lead to poor yield of the bacteria in cultures.

A simple modification of the candle jar technique could overcome these limitations. Placing a lighted candle inside a closed polycarbonate jar leads to reduction of oxygen by combustion. The residual 1-2\% oxygen in the air can be slowly purged out by including a plate containing reducing chemicals. Oxygen consumption by a lighted candle placed inside a closed jar till its point of extinction was assessed by a simple experiment by placing a burning candle under a water-sealed measuring cylinder. Considering the fact that some volume of $\mathrm{CO}_{2}$ was added due to the combustion it was established that 
only $1-2 \%$ of the oxygen content of the air inside the sealed cylinder remained unburned after candle extinction.

The remaining oxygen was then slowly absorbed by incorporating steel wool dipped in a solution of copper sulphate in sulphuric acid. One gram of activated steel wool absorbs approximately $1 \mathrm{ml}$ of oxygen per minute. ${ }^{[5,12]}$ So from a $1000 \mathrm{ml}$ sealed jar, $10-20 \mathrm{ml}$ oxygen remaining after candle extinction can be removed theoretically within 2-4 minutes, if $5 \mathrm{~g}$ of treated steel wool is used. The burning of the white wax candle in the candle jar system leads to generation of $4-5 \%$ $\mathrm{CO}_{2}$, which is beneficial for the growth of most medically important anaerobes. $\mathrm{A} \mathrm{CO}_{2}$ generator was also included in the system to elevate $\mathrm{CO}_{2}$ level in the jar to approximately $10 \%$. The stagnant air inside the Petri-plates would be replaced by heavier $\mathrm{CO}_{2}$, so that the air inside the Petri plates also gets reduced in the slow combustion process.[13]

The advantages of this system are that it is portable and compact and thus suitable for bedside inoculation of the samples, thereby eliminating the transport cost and delay. The minimum time that is required for inoculating and setting up the system is tolerable for most medically important anaerobes and thus will not reduce yield significantly. Moreover the compact system can be placed inside any regular incubator, so bulky apparatus are not required. The materials required are readily available and cheap and thus suitable for even small centres. The limitations of the system may include the production of heat and toxic gases such as carbon monoxide and sulphur dioxide due to combustion, which may hinder the growth of anaerobes.

But the heat generated is quite less and does not exceed the incubation temperature. Also by using a smoke free white wax candle, the toxic gas production can be minimized. Our study results also supported this aspect. Air tightness of the system must be ensured by placing anaerobic indicators, such as methylene blue in the jars. It is better that we use the cheaper, lower quality grease free iron wool that is not coated with rust proof alloys for efficient reaction. Also the copper sulphate solution was used within seven days of preparation for optimum reaction.

One of the most important requisites for anaerobic culture is the use of pre-reduced media. Even when oxygen is removed from the culture environment, unless the media are appropriately reduced before inoculation they have been observed to give poor growth due to the dissolved oxygen already present in the media. With the modified candle jar system, pre-reduced media can easily be obtained at no extra cost by simply placing the media in this system for 24-48 hours prior to inoculation.

In our innovative Modified Candle jar method, inoculation and incubation of sample starts at the bedside of the patient. The advantages over other anaerobic culture methods is that there is no requirement of Robertson's cook meat medium as a transport medium since direct inoculation starts at the bed side, thereby preventing toxic exposure to oxygen while transporting to the laboratory. Also the critical level of oxygen of $<1 \%$ is achieved instantly, making it superior to Gaspak in the recovery of obligate anaerobes.

Our technique is also better than the Anaerobic bag culture method, since here we use plastic jar which has a larger surface area as compared to that of the plastic bag, so that proper convection current will be maintained and there will be no trapped air present in between the petri plates and inside the media. One sample can be studied at a time.
It also provides good yield of growth as inoculation and anaerobiosis is initiated at collection point. Turnaround time from point of collection to complete identification and antibiogram is around five days, which is comparable with other methods of anaerobiosis.

In recent times, Metronidazole which used to be the drug of choice for anaerobic infections has shown development of resistance which is as high as $63 \% .[6,14-15]$ In our study also we got $33.33 \%$ resistance to metronidazole.

This method is cheaper and reproducible and thus can be incorporated in resource poor set up.

\section{REFERENCES}

1. Hall GS. Introduction. In HD Isenberg (ed.), Clinical Microbiology Procedures Handbook, Vol 1. Washington, DC. American Society for Microbiology 2007;4.1.1-4.1.2.

2. Finegold SM and George WL. Anaerobic Infections in Humans. Academic Press, Inc., San Diego, Calif 1989.

3. Maiti PK, Haldar J, Mukherjee P, Dey R. Anaerobic culture on growth efficient bi-layered culture plate in a modified candle jar using a rapid and slow combustion system. Indian Journal of Medical Microbiology. 2013;31(2):173176.

4. Cruickshank R, Duguid JP, Marimon BP, Swain RHA. Cultivation of Bacteria and Fungi: Culture in an Atmosphere with Added Carbon Dioxide. Medical Microbiology, Vol- II. $12^{\text {th }}$ ed. Churchill Livingstone, Hong Kong 1975; Page: 160.

5. Rosenblatt JE, Stewart PR. Anaerobic bag culture method. J Clin Microbiol 1975;1(6):527-30.

6. Bharadwaj R. Anaerobic microbiology: Time to rejuvenate. Indian J Med. Microbiol 2012;30(1):3-5.

7. Citron DM, et al. Evaluation of the E-Test for Susceptibility Testing of AnaerobicBacteria. J Clin Microbiol Oct. 1991;29(10):2197-2203.

8. CLSI. Performance standards for antimicrobial susceptibility testing; Twenty-fifth informational supplement. CLSI document M100-S-25. Wayne, PA: Clinical and Laboratory Standards Institute; 2015.

9. Spears RW, Freter R. Improved isolation of anaerobic bacteria from the mouse cecum by maintaining continuous strict anaerobiosis. Proc Soc Exptl Biol Med 1967;124: 903-9.

10. Aranki A, Syed SA, Kenney EB, Freter R. Isolation of anaerobic bacteria from human gingival and mouse cecum by means of a simplified glove box procedure. Appl Microbiol 1969;17:568-76.

11. Seip WF, Evans GL. Atmospheric analysis \& redox potentials of culture media in the gaspak system. J Clin Microbiol 1980;11:226-233.

12. Cheesebrough M. Culturing Bacterial Pathogens In: District Laboratory Practice in Tropical Countries Part2, 2 ${ }^{\text {nd }}$ ed. Cambridge University Press, Cambridge, UK. 2006.

13. Maiti PK. Anaerobic Culture on Modified Blood Agar Plate kept in a two steps combustion Candle Jar system. Patent Office Journal. 23/3/2012. Application no. 191/KOL/2012 A.

14. Raymundo M, Mendoza MT. The microbiologic features and clinical outcome of diabetic foot infections among patients admitted at UP -PGH. Phil J Microbiol Infect Dis 2002;31:54-63.

15. Lily SY $\mathrm{Ng}$, et al. Anaerobic culture of diabetic foot infections: organisms and Antimicrobial susceptibilities. Ann Acad Med Singapore. November 2008;37(11):936-9. 


\begin{tabular}{|c|c|c|c|c|c|c|}
\hline $\begin{array}{c}\text { Name of Anaerobic } \\
\text { Bacteria }\end{array}$ & Antibiotic & Sensitivity & (\%) & & \\
\hline $\begin{array}{c}\text { Bacteroides } \\
\text { fragilis (n=4) }\end{array}$ & $1(25 \%)$ & $3(75 \%)$ & $4(100 \%)$ & $4(100 \%)$ & $2(50 \%)$ & metronidazole \\
\hline penicillin & cefoxitin & clindamycin & imipenem & $\begin{array}{c}\text { chloramphenic } \\
\text { ol }\end{array}$ & $\begin{array}{c}\text { vancomyc } \\
\text { in }\end{array}$ \\
\hline $\begin{array}{c}\text { Peptostreptococcus } \\
\text { anaerobius (n=2) }\end{array}$ & $0(0 \%)$ & $0(0 \%)$ & $2(100 \%)$ & $2(100 \%)$ & $1(50 \%)$ & $2(100 \%)$ \\
\hline \multicolumn{7}{|c|}{ Table 1: Antibiotic Susceptibility Tests Result of Isolated } \\
Anaerobic Bacteria Detected by E Test Method
\end{tabular}

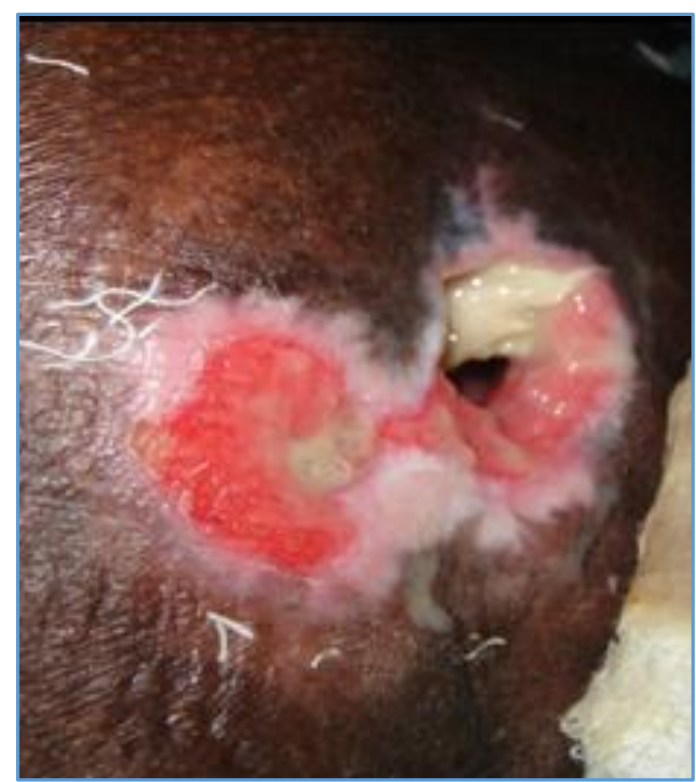

Fig. 1: Deep Seated Pressure Ulcers showing that the Ulcer Extends up to the Muscle

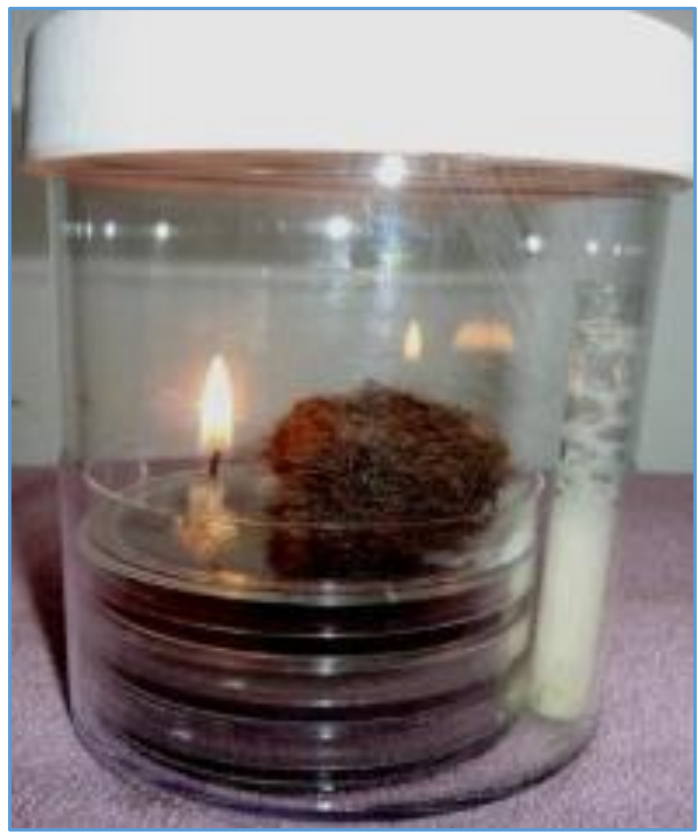

Fig. 2: Modified Candle Jar: Full Set Up with Brucella Blood Agar Plates and $\mathrm{CO}_{2}$ Generator in Test Tube

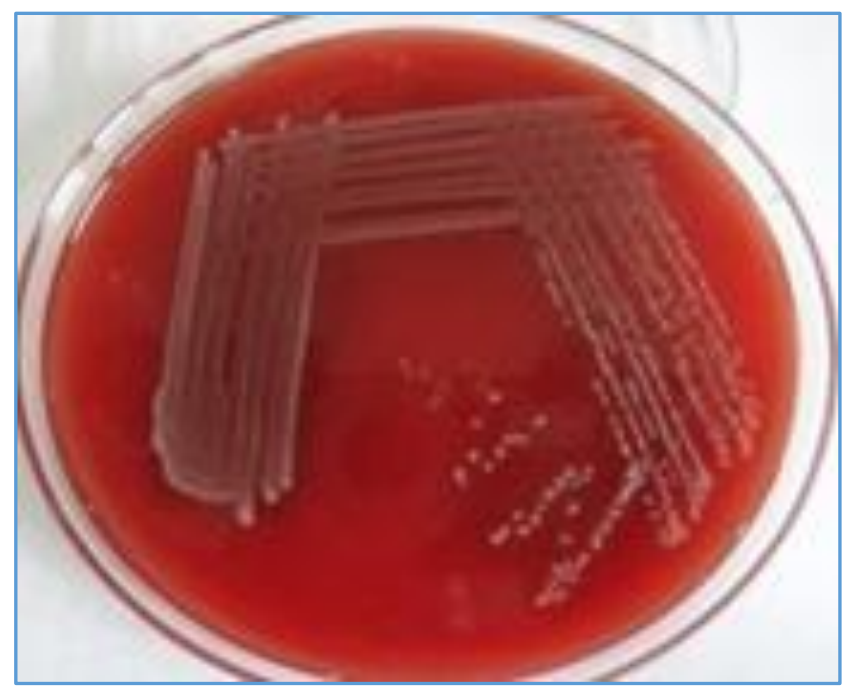

Fig. 3: (a): Growth of Bacteroids fragilis in Brucella blood agar

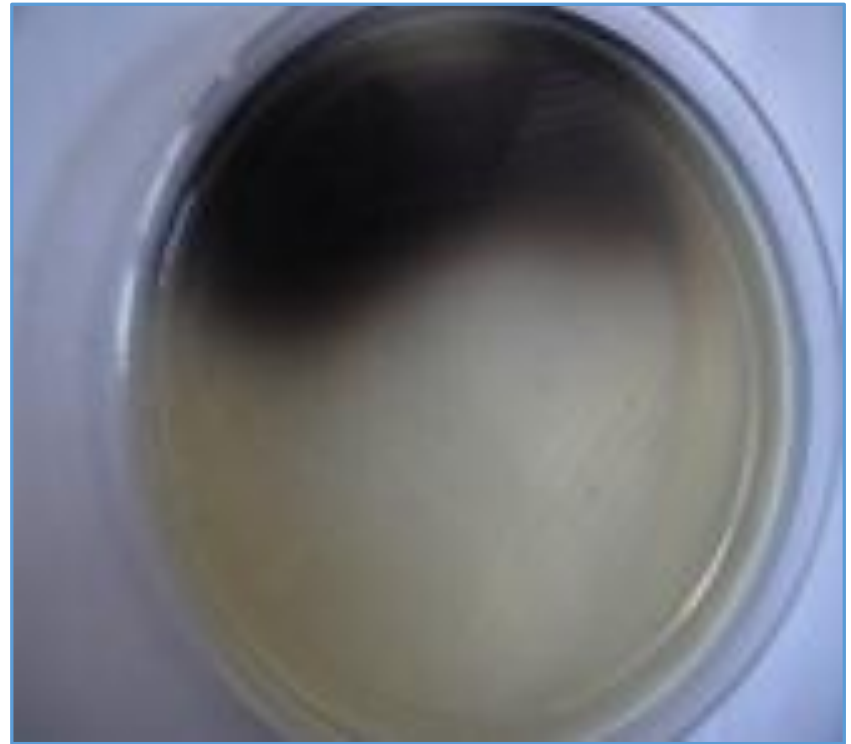

Fig. 3: (b): Growth of Black Colonies of Bacteroids fragilis in Bacteroides Bile Esculin agar 
Jemds.com

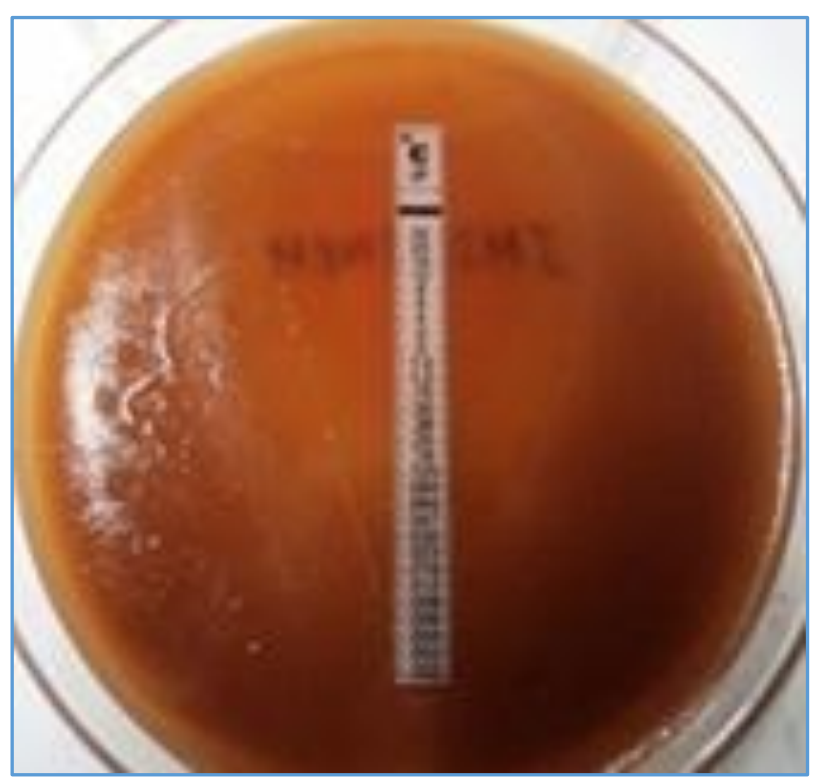

Fig. 4: Antibiotic Sensitivity Testing by E-Test Strips
Original Article

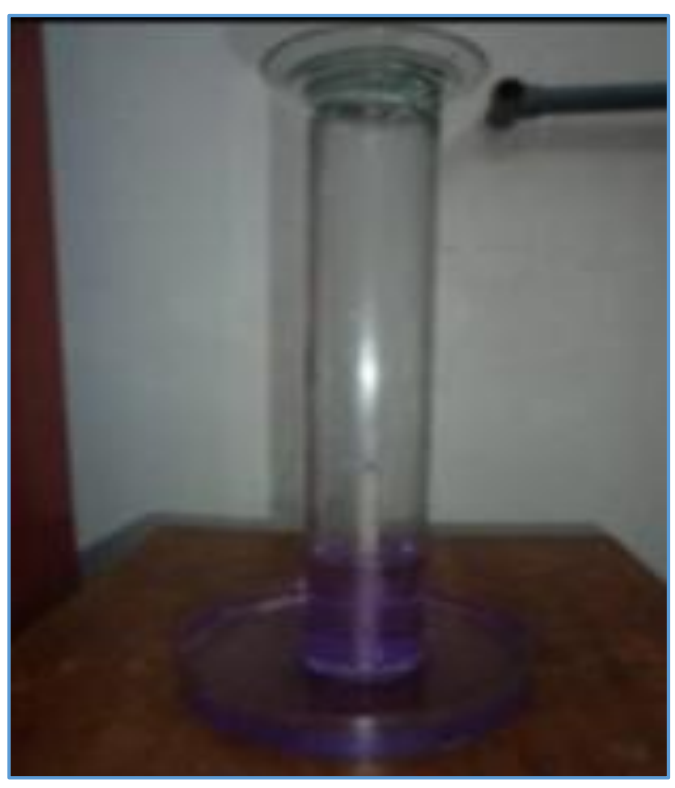

Fig. 5: Simple Experiment with Inverted Cylinder Placed Over Water with Lighted Candle to Determine Oxygen Consumption due to Candle Combustion 